\title{
Towards a Standardized Approach for Critical Care Nursing Practices: Development of Evidence Based Analgo-Sedation Guidelines for Adult Mechanically Ventilated Patients
}

\author{
Sahar Younes Othman ${ }^{1}$, Mona Mohamed EL-Hady ${ }^{2}$, \\ Mohamed Ahmed A. Sultan \\ ${ }^{I}$ Critical Care and Emergency Nursing, Department of Critical Care and Emergency Nursing, Faculty of \\ Nursing, Damanhour University, Egypt \\ ${ }^{2}$ Critical Care and Emergency Nursing, Department of Critical Care and Emergency Nursing, Faculty of \\ Nursing Mansoura University, Egypt \\ ${ }^{3}$ Anesthesia and Intensive Care, Department of Anesthesia and Intensive Care, Faculty of Medicine, Mansoura \\ University, Egypt
}

\begin{abstract}
:
Background: The judicious and appropriate use of analgesic/sedative medications is an essential goal of providing safe and effective nursing management for mechanically ventilated patients. One method of achieving this goal is the use of evidence based guidelines. Although numerous researches have been conducted to explore the clinical benefits of nurse managed-sedation protocol/guidelines, none of them was conducted in Egypt.

Aim: This study aimed to develop evidence based analgo-sedation guidelines for adult mechanically ventilated patients.

Methods: A methodological research design was utilized according to the Scottish Intercollegiate Guidelines Network (SIGN) process of guidelines development. The present study was conducted on three phases i.e. observation phase, developing guidelines phase and guidelines appraisal phase. The observation phase was conducted on forty patients to better understand how routine analgesia/sedation practices provided by critical care nurses (CCNs) influenced patients' clinical outcomes. Also, the results of this phase were served as a basis for the elaboration of the intended analgo-sedation guidelines. The study was conducted at trauma adult ICU of Emergency Hospital of Mansoura University, Egypt. Two tools were used for data collection: Analgesia/sedation assessment record and Appraisal of Guidelines for Research and Evaluation (AGREE) II instrument that was used for appraising the developed guidelines.

Results: Unsatisfactory nursing analgesia/sedation practices were found during the observational phase which had negative effects on patients' clinical outcomes. In response to these findings, the authors developed analgosedation guidelines that included fifty recommendations. The overall quality of intended guidelines was relatively good. The majority of academic nursing appraisers as well as two thirds of academic medical appraisers recommended the current analgo-sedation guidelines to be used in practice without modifications. Conclusions: The observational phase findings of the current study provide additional rationale for the application of standardized approach in analgesia/sedation management for mechanically ventilated patients. The developed guidelines may be a first step in a patient-safety initiative for sedating mechanically ventilated patients in Egypt. It was strongly recommended by the appraisers to incorporate these guidelines in clinical practice as a routine of unit care.
\end{abstract}

Keywords: Analgesia, Critically ill, Guidelines, Mechanical ventilation and Sedation

\section{Introduction}

Most critically ill patients in the intensive care units (ICUs) often experience pain, anxiety and agitation, particularly in the mechanically ventilated patients. ${ }^{[1]}$ The Payen et al. (2007) study has shown that $33 \%$ of the mechanically ventilated patients experienced pain at rest and $56 \%$ experienced pain during a procedure. ${ }^{[2]}$ Also anxiety is commonly reported with an incidence that ranges from $30.8 \%$ to $80 \%{ }^{[3,4]}$ These problems are mostly caused by the patient's underlying illness, surrounding medical devices, unfamiliar environment, invasive interventions, communication barriers and desynchronization with the ventilator. ${ }^{[1,5]}$ Untreated pain and anxiety can lead to an increasing in the sympathetic stress response that includes increased endogenous catecholamine activity, increased oxygen consumption, tachycardia, hypercoagulability, hypermetabolism, and immunosuppression ${ }^{[6]}$ Furthermore, unrelieved pain and anxiety can lead to severe agitation that may aggravate the risk of adverse events such as accidental self-extubation, loss of venous catheters, and self-injury or injury to the clinicians. Therefore, optimizing analgesia and sedation is an universal goal for critical care practitioners. ${ }^{[7]}$ 
Critically ill patients are routinely provided analgesia and sedation to prevent pain and anxiety, permit invasive procedures, reduce stress and oxygen consumption, improve synchrony with mechanical ventilation (MV) and also to facilitate nursing care ${ }^{[8-12]}$ Lower oxygen demand of mechanically ventilated patients is associated with successful ventilator weaning. The early weaning of MV can reduce the risk of complications of MV, e.g. ventilator-associated pneumonia. As a result, it shortens the ICU length of stay (ICU LOS). In short, the use of analgesia and sedation can be considered to improve the quality of life of mechanically ventilated patients in their hospitalization. ${ }^{[1,7]}$ On the other hand, over-sedation is associated with worse clinical outcomes, including longer time on MV, prolonged ICU LOS, increased risk of pneumonia and sepsis, increased brain dysfunction (delirium and coma), and increased mortality and costs. ${ }^{[13]}$

An important part of nursing care in the ICU is providing proper sedation for patients receiving MV and providing patient's comfort without coma. ${ }^{[14]}$ Sedation and pain relief require not only interdisciplinary assessment and decision-making, but also independent assessment and decision-making by (CCNs). The CCNs play a key role in assessing patients' need of sedation, as well as determining the dosage and frequency in titrating analgesia and sedatives within prescribed limits which presents a significant challenge to them. ${ }^{[1,10,14]}$ Furthermore, the use of non-pharmacological interventions (relaxation, comfortable rest in bed, removal of noise, repeated information in short form, the presence of significant others, light, music and mobilization) are essential for patient's safety and comfort, and therefore constitute a major part of the CCNs' work. Since analgesics and sedatives in ICUs are drugs with potentially serious side effects, nurses bear a large responsibility in maintaining and developing the quality of pain treatment and sedation. ${ }^{[8]}$ Unfortunately, studies have indicated that nurses underestimate pain and the level of sedation and they do not assess pain and sedation levels in critically ill patients by routine methods. ${ }^{[5,7,10]}$ Sedatives and analgesics are formally prescribed by physicians and administered by nurses, often with a wide margin of discretion in dose and without explicit understanding of the target level of sedation. ${ }^{[15]}$

Sedation of critically ill patients is challenging, and current literature and guidelines have contributed to a significant shift in strategies for these patients. Critically ill patients have unique metabolic demands, and patient-specific factors such as organ dysfunction and age must be considered when creating patient-centered sedation plans. ${ }^{[16]}$ Various strategies have been proposed to improve sedation management of critically ill patients: sedation assessment instruments; sedation guidelines, algorithms or protocols to guide assessment and therapy; implementation of daily sedation interruptions; targeting minimal levels of sedation and regular assessment of sedation and analgesia requirements. Also, analgo-sedation is a prevailing sedation strategy that focuses first on the relief of pain and is associated with improved patient outcomes. ${ }^{[6,12,17]}$ The importance of balanced sedation and pain treatment in ICUs is evident, but regimes and use of medication differ widely and require improvement. ${ }^{[18-21]}$

Patients receiving MV is an area of healthcare that warrants investigation due to the opportunity to improve both clinical outcomes and the economic costs of care. Despite all the available evidence, best sedation practices are still heterogeneous and insufficiently implemented worldwide. It is imperative to address the obvious gap between research and practice. More data are needed to help in establishing the evidence based nursing practices and providing the best care to mechanically ventilated patients. ${ }^{[22]}$ Previous studies have focused on the use of various sedative and analgesic medications and regimes. What has not been explored is the process by which CCNs and physicians assess patients' sedation needs and work together toward a defined level of sedation and pain for the ICU patient. ${ }^{[18]}$

The judicious and appropriate use of sedative medications is an essential goal of providing safe and effective sedation nursing management for mechanically ventilated patients. One method of achieving this goal is the use of evidence based clinical practice guidelines which incorporate sedation scales for the maintenance of analgesia and sedation in ICU patients. ${ }^{[1]}$ Clinical practice guidelines (CPGs) are systematically developed statements designed to assist practitioner and patient decisions about appropriate healthcare for specific clinical circumstances. Unlike protocols, policies, clinical pathways and standards of care, CPGs are designed to assist decision making rather than prescribe, mandate or give authority for an aspect of care or procedure. The Institute of Medicine in the USA has suggested that the use of the term 'guideline' should be restricted to systematically developed advisory statements which have been created according to validated methodologies. In addition, valid evidence based CPGs should integrate the expertise of a multidisciplinary group of clinicians and the best available research evidence, to make recommendations that support clinical decision-making. ${ }^{[11,23]}$

\subsection{Significance of the Study}

In the local study setting, there are no standardized sedation guidelines or protocols being used in the ICU. The management of sedation therapy often differs among ICU physicians. There are also different preferences of the physicians regarding to the types of sedatives or analgesics used. It is documented that standardized analgo-sedation practices (including sedation protocols, algorithms and guidelines) can improve the patients' clinical outcomes. ${ }^{[1,8,9,11-13,15,17]}$ Beside its benefits on patients' clinical outcomes, it can also facilitate decision 
making of $\mathrm{CCNs}$ in sedation titration and minimize the variations of practice to assure a systemic approach in nursing care. More importantly, the use of analgo-sedation guidelines is not only advantageous for the critically ill patients, it would also be beneficial for the unit and the hospital in terms of cost saving. It is well known that MV is a high-cost intervention. The use of effective analgo-sedation guidelines is expected to reduce the dosage of sedatives/analgesics and the complications among mechanically ventilated patients. Consequently, it reduces the total costs of health care. ${ }^{[1]}$

Although numerous researches have been conducted to explore the clinical benefits of nurse managedanalgo-sedation protocol/guidelines, none of them was conducted in Egypt. The study by Brook et al. (1999) ${ }^{[24]}$ and Elliott $(2005)^{[11]}$ were conducted in North America and Australia. There are major cultural differences between CCNs practices in North America, Australia and Egypt, e.g. legal requirements for the prescription of medications, the model of care and undergraduate nursing education are all different. Having considered the possible serious consequences of inappropriate sedatives/analgesics administration and inadequate Egyptian researches available in this regard, there is an urging need to develop evidence based analgo-sedation guidelines that may be used by Egyptian CCNs.

\subsection{Aim of the study}

The aim of the study is to develop evidence based analgo-sedation guidelines that will assist CCNs to intervene consistently with adult mechanically ventilated patients.

\subsection{Research questions}

1.3.1 Is there a standardized nursing approach for analgesia/sedation of adult mechanically ventilated patients being used in the selected ICU setting?

1.3.2 What are the patients' clinical outcomes of routine CCNs' practice of analgesia and sedation?

1.3.3 What are the recommendations for analgesia and sedation of adult mechanically ventilated patients?

1.3.4 What are the appraisers opinions of the analgo-sedation guidelines developed in the current study?

\subsection{Design}

\section{Subjects and Methods}

A methodological research design was used to achieve the aim of the current study.

\subsection{Setting}

The study took place in a sixteen-bed trauma adult ICU at Emergency Hospital of Mansoura University, Egypt. In this unit, the ratio of registered nurse to patient is 1:2 for mechanically ventilated patients with the additional clinical support of an internship student from faculty of nursing. The unit has four resident physicians who are on call 24 hours a day. Registered nurses perform all the nursing care for ICU patients.

\subsection{Subjects}

Convenience sampling technique was used for patient recruitment during the current study. Forty patients admitted to the predetermined setting were enrolled in the study. The inclusion criteria were as follows: male and female patients older than 18 years; patients received invasive MV for more than 48 hour; patients had Glasgow Coma Scale (GCS) of higher than 7; and patients required intravenous sedation for more than 24 hours. Exclusion criteria included the following: a second period of MV during hospitalization, admission following cardiac arrest, ICU readmission, drug abuse and short-term expected mortality.

Sample calculation method: After application of inclusion criteria, the average number of beds included 4 with an average hospital stay of 7 days i.e. 16 beds in each month. The study was conducted over a period of 3 months, so 48 beds are eligible to be included during this 3 month period. We followed up 40 beds as the others lost follow up. So the response rate is (40 out of 48 ) was $83.3 \%$.

\subsection{Tools for data collection:}

Two tools were used to collect the necessary data of the current study.

\section{Tool one: Analgesia/sedation assessment tool}

It was combined by the researchers after reviewing the related literature ${ }^{[25-27]}$ to assess, monitor and document data of the studied patients during analgesia/sedation routine nursing practices throughout the study period. It consists of three parts:

Part (1): Patient's Profile: This part was developed by the researchers to collect data about patient's sociodemographics and baseline characteristics including (gender, age, diagnosis, past history, indication of MV, GCS and Acute Physiology and Chronic Health Evaluation APACHE II score). Furthermore, it includes patients clinical outcomes of analgesia/sedation routine nursing practices including; duration of MV; ICU LOS and adverse events such as (self-extubation, catheter removal, weaning failure and if the patient diagnosed as VAP).

Part (2) Richmond Agitation Sedation Scale (RASS): This scale was adopted from Sessler et al. (2002) and used by the researchers to assess patients' sedation/agitation level. RASS is a 10-point scale with four levels for 
agitation (+1 to +4 "combative"), one level to denote a calm and alert state $(0)$, and 5 levels of sedation $(-1$ to $-5) .{ }^{[26]}$

RASS scoring system:

Patients in a RASS range of -2 to +1 is considered lightly sedated, RASS range of -3 to -5 deeply sedated and $>2$ agitated. ${ }^{[26]}$

Part (3) Critical-Care Pain Observation Tool (CPOT): This scale was adopted from Gelinas et al. (2008) and used by the researchers to assess patient's pain level. The CPOT consists of 4 domains: facial expression, body movement, muscle tension, and compliance with the ventilator. Each domain is scored from 0 to 2, total scores range from 0 (no pain) to 8 (most pain). ${ }^{[27]}$

CPOT scoring system:

Patients in a CPOT range of 1 to 3 is considered mild pain, CPOT range of 4 to 5 moderate pain and $\geq 6$ sever pain. ${ }^{[27]}$

Part 2 and 3 of the analgesia/sedation assessment tool were designed in a flow sheet which involves data from patient's admission till discharge. In addition the type and amount of sedatives/analgesics administered are included.

\section{Tool two: Appraisal of Guidelines for Research and Evaluation (AGREE) II instrument}

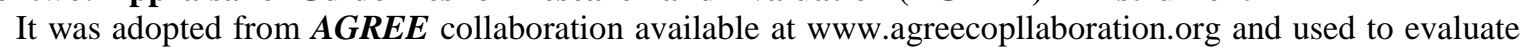
the developed analgo-sedation guidelines by nursing and medical specialists. AGREE II instrument consists of $23 \mathrm{key}$ items organized in six domains followed by two global rating items (Overall Assessment). Each domain is intended to capture a separate dimension of guidelines quality. ${ }^{[28]}$

- Domain 1. Scope and purpose is concerned with the overall aim of the guidelines, the specific health questions, and the target population (items 1-3).

- Domain 2. Stakeholder involvement focuses on the extent to which the guidelines were developed by the appropriate stakeholders and represents the views of its intended users (items 4-6).

- Domain 3. Rigour of development relates to the process used to gather and synthesize the evidence, the methods to formulate the recommendations, and to update them (items 7-14).

- Domain 4. Clarity of presentation deals with the language, structure, and format of the guidelines (items 1517).

- Domain 5. Applicability pertains to the likely barriers and facilitators to implementation, strategies to improve uptake, and resource implications of applying the guidelines (items 18-21).

- Domain 6. Editorial independence is concerned with the formulation of recommendations not being unduly biased with competing interests (items 22-23).

- Overall assessment includes the rating of the overall quality of the guidelines and whether the guidelines would be recommended for use in practice. This contains a series of options yes recommend, yes recommend with modifications and not recommend.

\section{Rating scale of AGREE II instrument}

Each of the AGREE II items are rated on a 7-point scale (1-strongly disagree to 7-strongly agree).

- Score of 1 (Strongly disagree): A score of 1 should be given when there is no information that is relevant to the AGREE II item or if the concept is very poorly reported.

- Score of 7 (Strongly agree): A score of 7 should be given if the quality of reporting is exceptional and where the full criteria and considerations articulated in the User's Manual have been met.

- Scores between 2 and 6: A score between 2 and 6 is assigned when the reporting of the AGREE II item does not meet the full criteria or considerations.

\subsection{Validity and reliability of study tools}

Content validity of the current study tools was ensured by having a panel of five experts that have experience in instrument development, critical care nursing, and the performance of sedation. The panel of experts reviewed the instrument and confirmed that the individual items included were appropriate, accurate, and representative of the content domain being evaluated within the study. The reliability and validity of RASS was acceptable in another research done by Mansouri et al. (2013), the Pearson correlation coefficients obtained was $0.92 .{ }^{[29]}$ Of the available observational pain scales, the CPOT has shown superior reliability and validity when used in nonverbal critically ill adults. The interrater reliability of CPOT was assessed by Gélinas and Johnson (2007) and found to be 0.80-0.93. ${ }^{[30]}$ Also, the AGREE II tool has been tested for reliability and validity, and is applicable to a wide variety of health professionals. ${ }^{[31]}$

\subsection{Pilot study}

A pilot study was conducted after the development of the study tools and before starting data collection on $10 \%$ of the study sample who were excluded from the total sample. The aim of the pilot study was to determine the clarity and applicability of the designed tools and accordingly necessary modifications were done. 


\subsection{Field work and data collection}

This methodological study was conducted from March 2015 to April 2016 based on the criteria of the Scottish Intercollegiate Guidelines Network (SIGN) ${ }^{[32]}$ on three phases as demonstrated in figure 1.

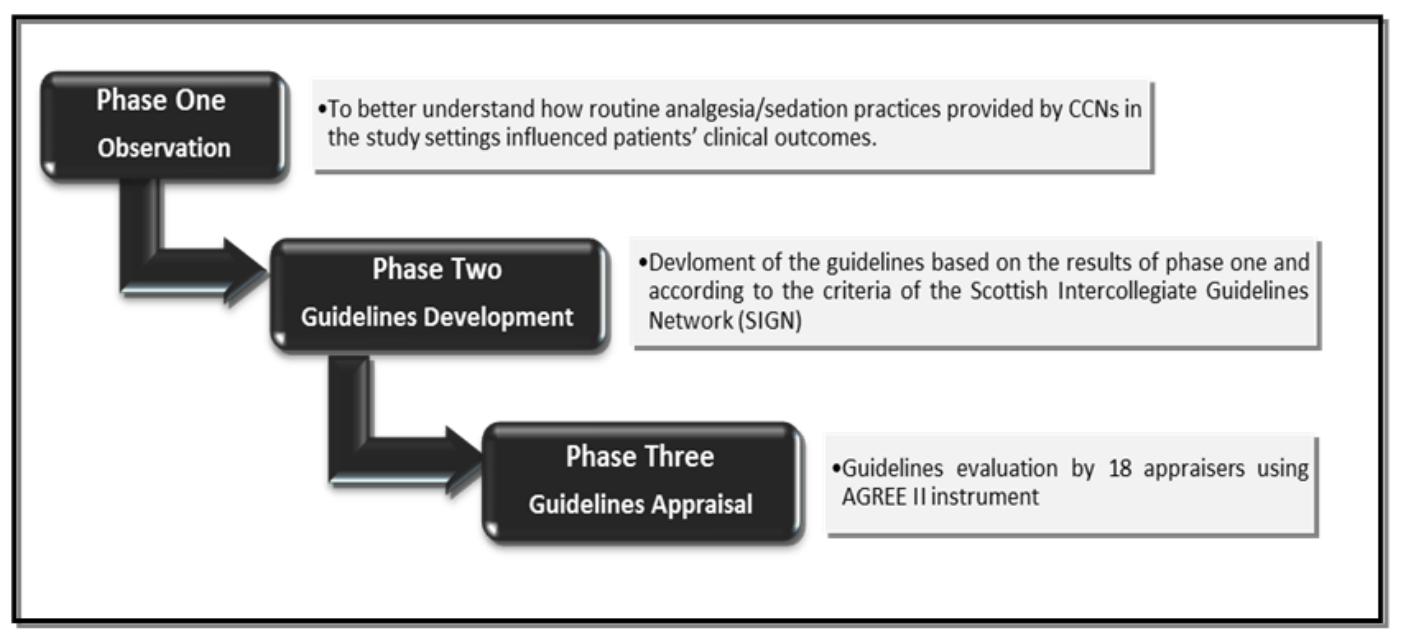

Figure 1 Study flow chart

\subsubsection{Phase one: "Observation Phase"}

- Over a 3-month period, the researchers began to better understand how routine analgesia/sedation practices provided by CCNs in the study setting influenced patients' clinical outcomes. From March 2015 till May 2015, all eligible patients (40 patients) in the intended ICU were followed up by the researchers from the time of enrollment in the study until discharge or death.

- For each patient, socio-demographics and baseline clinical data were collected on admission and documented by the researchers in tool one part (1). Then registration of patients' pain level using (CPOT) and sedation level using (RASS) was conducted every 4 hours (Part $2 \& 3$ of tool one). Sedative and analgesic dosages (intravenous infusion or bolus) and reasons for administration were documented daily by the researchers.

- The studied patients were observed daily for the appearance of any adverse event (self-extubation, catheter removal, weaning failure and if the patient diagnosed as VAP) using part (1) of tool one. Unscheduled selfextubation was defined as the extubation or decannulation of a tracheostomy which was unplanned and facilitated by the patient. Also, the incidence of adverse event is defined by the proportion of patients who developed at least one adverse event during his/her ICU stay.

- Patient's secondary clinical outcomes were obtained by using measurable indicators such as duration of MV and ICU LOS. These data were also recorded in part (1) of tool one. The duration of MV in days was defined as the initial time when the mechanical ventilator was applied until the ventilator is discontinued. The ICU LOS in days was calculated by determining the date and time of ICU admission and the date and time of transfer out of the ICU or death, and summing the total number of days.

\subsubsection{Phase Two: Analgo-sedation guidelines development}

The intended analgo-sedation guidelines were developed according to the criteria of the Scottish Intercollegiate Guidelines Network (SIGN). ${ }^{[32]}$ The steps of guidelines development were as following:

\subsubsection{Determination of needs and scope of the guidelines}

The need and scope of the current analgo-sedation guidelines were ascertained through the identification of patients' outcomes of routine analgesia/sedation practices provided by CCNs in the study setting which obtained from phase one. Also the need for these guidelines was obtained from the recommendations of the national researches on sedation practices which conducted in Egypt. ${ }^{[33,34]}$ The observation phase of the current study provide some insight into nursing sedation management as well as a brief overview of the unit characteristics and resources that helped in the guidelines development. It was observed that there was no explicit analgosedation protocols/guidelines used in the intended ICU. Analgesia/sedation practices were carried out in an empirical manner, differing according to physician preference and driven by the availability of medical staff.

\subsubsection{Establishment of a multidisciplinary working group}

In addition to the researchers, five academic experts in critical care nursing; critical care medicine, and development of evidence-based guidelines were convened to review the process of guidelines development and its primary drafts. 


\subsubsection{Defining the purpose and the target audience for the guidelines}

As a reflection of the multidisciplinary nature of the sedation practice the guidelines were aimed at both medical and nursing staff. The aim was to standardize the team approach and keep the sedation practices patient centered.

\subsubsection{Stating clinical search questions}

Nine clinical search questions were constructed using the PICO format $(\mathrm{P}=$ patient, $\mathrm{I}=$ intervention, $\mathrm{C}=$ comparison, $\mathrm{O}=$ outcome). Concise PICO statements or questions help to target the search of the evidence. ${ }^{[35]}$ See box (1).

\section{Box1. Clinical search questions}

1. How nurses and physicians manage and describe sedation for mechanically ventilated adult patients?

2. Should pain/agitation assessment be routinely performed in adult ICU patients?

3. What are the most valid and reliable pain/sedation scales in mechanically ventilated adult ICU patients?

4. Should physiological parameters (vital signs) be used to assess pain in adult ICU patients?

5. Should procedure-related pain be treated pre-emptively in adult ICU patients?

6. What types of medications should be administered for pain relief and sedation in adult ICU patients?

7. Should analgesia-first sedation (i.e., analgo-sedation) or sedative-based sedation be used in mechanically ventilated ICU patients?

8. Should sedation protocol/ algorithm be used in adult mechanically ventilated patients?

9. Should non-pharmacologic interventions be used to promote sleep and comfort in adult mechanically ventilated patients?

\subsubsection{Searching for the evidence.}

A literature search was undertaken to identify potentially relevant evidence to develop the intended evidence-based guidelines. Review of the literature was conducted from 1999 up to 2015 from eight electronic bibliographic databases: PubMed at: http://www.pubmed.gov, Science direct at: http:// www.sciencedirect.com, MEDLINE, Cochrane Database of Systematic Reviews at: http://www.cochranelibrary.com, National Guidelines Clearing house at www.guidelines.gov, Scottish Intercollegiate Guidelines Network at: www.sign.ac.uk, National Institute for Health and Clinical Excellence (NICE) at www.nice.org.uk, and Center for Review and Dissemination (CRD) at www.york.ac.uk/ins/crd/). Search parameters included published English-only manuscripts on adults $(>18 \mathrm{yr})$. Studies with less than 30 patients, editorials, narrative reviews, case reports, animal or in vitro studies, and letters to the editor were excluded. The key words (sedation, analgesia, analgo-sedation, protocol, guidelines, evidence based, intensive care unit, critically ill, and mechanical ventilation) were used to search for relevant literatures.

\subsubsection{Evaluating evidence and grading recommendations}

The quality of each individual study was rated by two members of guidelines developers team using the SIGN coding system (Scottish Intercollegiate Guidelines Network, 2008). ${ }^{[32]}$ The two members independently completed evaluation of evidence and grading recommendations and summarized the findings of each study and evaluated the quality of evidence. The SIGN system included three main steps to evaluate evidence and grade the guidelines recommendations, namely; study validity rating, determination level of evidence and finally the grade of recommendation.

\subsection{First step: study validity rating}

All primary studies addressing the relevant topic were appraised by using SIGN checklist that was appropriate to the study design, and then were individually rated for internal validity using the system that is shown in box 2.

Box 2. Rating of the internal validity for studies according to the (SIGN) System

Rating Description

$++\quad$ All or most of the criteria have been fulfilled

$+\quad$ Some of the criteria have been fulfilled

- $\quad$ Few or no criteria fulfilled 


\subsection{Second step: determination of evidence level:}

The study design was assigned by numerical prefix using the system below (Box 3): Then each study was assigned to a level of evidence by using the system below (Box 4):

\begin{tabular}{|c|c|}
\hline Numerical prefix & Study designs \\
\hline 1 & For systematic review or meta-analysis or randomized control trials (RCTs) \\
\hline 2 & For cohort and case control studies \\
\hline 3 & For case report series \\
\hline 4 & For expert opinion/logical argument/common sense \\
\hline
\end{tabular}

\begin{tabular}{|c|c|}
\hline $\begin{array}{c}\text { Box 4. The level of evidence system according to the (SIGN) System } \\
\text { T++ }\end{array}$ & $\begin{array}{l}\text { Type of Evidence } \\
\text { High quality meta-analysis systematic reviews of randomized control trials with } \\
\text { a very low risk of bias. }\end{array}$ \\
Well conducted meta-analysis systematic reviews or randomized control trials \\
with a low risk of bias. \\
Meta-analysis, systematic reviews, or randomized control trials with a high risk \\
of bias.
\end{tabular}

\subsection{Third step: grade of recommendation}

The detailed results of each study were considered in the formulation of each guidelines recommendation which was then graded using the following system (Box 5).

\begin{tabular}{|c|c|}
\hline Grade & Recommendation \\
\hline $\mathbf{A}$ & $\begin{array}{l}\text { At least one meta-analysis, systematic review, or RCT rated as } 1++ \text {. And } \\
\text { directly applicable to the target population, or a body of evidence consisting } \\
\text { principally of studies rated as } 1+\text {, directly applicable to the target population } \\
\text { and demonstrating overall consistency of results }\end{array}$ \\
\hline B & $\begin{array}{l}\text { A body of evidence including studies rated as } 2++ \text {, directly applicable to the } \\
\text { target population and demonstrating overall consistency of result, or } \\
\text { extrapolated evidence from studies rated as } 1++ \text { or } 1+\end{array}$ \\
\hline $\mathbf{C}$ & $\begin{array}{l}\text { A body of evidence including studies rated as } 2+\text {, directly applicable to the } \\
\text { target population and demonstrating overall consistency of result or } \\
\text { extrapolated evidence from studies rated as } 2++\end{array}$ \\
\hline D & Evidence level 3 or 4 , or extrapolated evidence from studies rated as $2+$ \\
\hline
\end{tabular}

\subsubsection{Formulation of guidelines drafts}

A draft of the intended guidelines including analgo-sedation algorithm was drawn up (See figure 2 analgosedation algorithm). The consensus of guidelines recommendations was reached using Delphi technique. The guidelines developers met three times over a period of 6-weeks. The guidelines were redrafted twice before the final agreed format was ready for piloting on the intended ICU.

\subsubsection{Phase Three: Guidelines appraisal}

The final analgo-sedation guidelines format was evaluated by 18 appraisers using AGREE II instrument (tool two) to measure its content validity and applicability. The appraisers group involved academic nursing staff members $(n=7)$, academic medical staff members $(n=3)$, practitioner CCNs $(n=4)$ and practitioner physicians $(n=4)$ who are working in adult ICU. The academic staff members were specialists in critical care nursing, anesthesiology and who had experience in evidence-based medicine and guidelines development and evaluation. 


\section{Scoring the AGREE II instrument}

i. Calculating domain quality scores: A quality score was calculated for each of the six AGREE II domains by the following formulas:

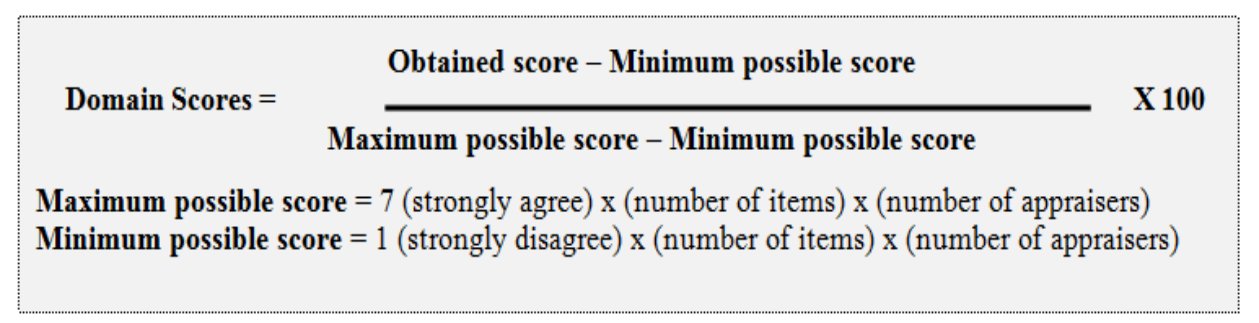

ii. Overall Assessment of analgo-sedation guidelines: Two overall assessment items required the appraisers to make a judgment as to the global quality of the guidelines. The appraisers gave an overall assessment of the guidelines from 1 (lowest) to 7 (highest) and were asked to state if they would recommend the guidelines, recommend it with modifications or not recommend it (using Tool two). Also, they were asked to provide comments to justify their rating. The appraisers did not communicate with each other during the appraisal process.

\subsubsection{Guidelines dissemination}

Prior piloting the intended guidelines, education sessions were scheduled over a two weeks period to inform nursing staff about the analgo-sedation guidelines and the process of its utilization.

\subsubsection{Pilot study}

A pilot study was conducted on five patients over a two weeks period to test the feasibility and content validity of the proposed analgo-sedation guidelines. Minor revisions were made to the guidelines in response to feedback from staff.

\subsection{Administrative and ethical considerations}

Permission was granted by the hospital administration and ICU director to conduct this study. Also approval was obtained from the Ethics Committee of the Faculty of Nursing, Mansoura University. All patients were assigned a code number to ensure confidentiality. The researchers consulted the patients' relatives for information about his/her opinion on patients' participation in the study and informed oral consent was obtained from them. Patients' relatives were also assured that if they did not wish to participate in the study, this would have no effect on the quality of care provided. The patients' anonymity and privacy were assured.

\subsection{Statistical analysis}

Data were entered and statistically analyzed using the Statistical Package for Social Sciences (SPSS) version 20. Following data entry, checking and verification processes were carried out to avoid any errors during data entry. Qualitative data were described as numbers and percentages. Quantitative data were tested for normality by Shapiro-Wilk test. The normally distributed variables were described as means (SD); while the non-normally distributed variables were described as medians (range).

\section{Results}

Results of the present study consist of three parts: firstly needs assessment of the studied patients; secondly guidelines' recommendations and thirdly the results of guidelines appraisal.

\subsection{Part I: Results of needs assessment of the studied patients}

This part of study results concerned with the studied patients' clinical outcomes of routine CCNs' practices regarding analgesia and sedation.

As shown in table 1, there were more men $(65 \%)$ than women in the study sample $(35 \%)$ and the mean age of the patients was $37.1 \pm 1.35$ year. Seventy percent of patients were admitted post trauma and $30 \%$ were admitted for other post-operative and medical diagnoses. Sixty percent of studied patients had no history of medical problems, $30 \%$ of them had respiratory and neurological disorders and only $10 \%$ had a history of cardiovascular disorder. The reason for MV was neurological disorder in $57.5 \%$ of the studied population. The mean APACHE II score on admission was $26.57 \pm 4.9$ while GCS was 9.5 \pm 2.2 .

As presented in table 2 the total mean scores of Agitation Sedation Scale (RASS) for the studied patients on admission were $0.77 \pm 2.7$. More than half of the patients $(55 \%)$ at this time were in a combative state (RASS score +1 to +4 ) while on discharge, $75 \%$ of them were in sedated state (RASS score -1 to -5 ). Figure 3 demonstrates changes in the RASS scores over the study period. It was found that the RASS scores for studied sample were fluctuated but remained within sedated level (between -1 to -4 ) all over the study period. It can 
observed from the same figure that during fifth and sixth days, the studied patients were observed to be in restless stat $(\mathrm{RASS}=+1)$.

As illustrated in table 3 and figure 4, on admission time the majority of patients $(52.5 \%)$ in the studied population had moderate pain (CPOT 4-5), $25 \%$ of patient were almost pain-free (CPOT 0), $20 \%$ experienced severe pain (CPOT 6-8), and only $2.5 \%$ had mild pain (CPOT 1-3). While on discharge less than half of studied patients $(47.5 \%)$ had moderate pain. It was observed that the average mean scores of Critical-Care Pain Observation Tool (CPOT) on admission were $(3.55 \pm 2.28)$ which were slightly reduced to be $(3.025 \pm 3.01)$ on discharge. As presented in figure 4, pain score for the whole studied sample was remained within moderate level all over the study period.

Drugs that were used to treat pain and agitation are shown in table 4. According to the findings, the mean doses of fentanyl for control of pain were $(4.12 \pm 6.34 \mathrm{mg} / \mathrm{day})$ in the studied patients. Fentanyl was the only prescribed analgesic and propofol and midazolam were the most commonly used sedatives during the study period. The results reflect high mean dose of sedatives was used to sedate the patients $(520.82 \pm 614.72$, $2880 \pm 960,1778.82 \pm 5804.1$ for midazolam, propofol and precedex respectively).

Table 5 shows occurrence of adverse events throughout the study period. Regarding the number of unintentional device removal, 55\% of studied patients removed their endotracheal tubes (ETT) at least once during their stay. About $47.5 \%$ and $40 \%$ of them had an accidental removal of urinary catheter and peripheral venous line respectively. After extubation, re-intubation occurred in $55 \%$ of the patients. The percent of tracheostomies performed while the patients were cared for in the study ICU was $27.5 \%$. Physical restraint was performed in about $55 \%$ of studied sample. Fifty percent of the studied patients were subjected to weaning failure and $32.5 \%$ of them diagnosed as ventilator-associated pneumonia.

Table 6 displays secondary outcomes throughout the study period. It was observed that the mean duration of sedation/analgesia was 5.25 \pm 2.18 days among the whole study sample. Mean duration of MV was 5.32 \pm 2.24 days. Furthermore, mean ICU length of stay was $6.07 \pm 2.58$ days.

\subsection{Part II: Analgo-sedation guidelines' recommendations}

\subsubsection{Pain assessment recommendations:}

On the basis of one RCT study (Level of evidence $1^{+ \text {)[24] }}$, three prospective cohort studies[36-38] (Level of evidence $2++$ ) and well-developed sedation guidelines ${ }^{[39]}$, it was concluded that pain assessment for all adult ICU patients should be performed regularly (Recommendation number 1.1 Box 6). The results of these studies revealed that regular pain assessment in adult ICU patients is associated with improved clinical outcomes. Pain assessment, especially if protocolized, has been significantly associated with a reduction in the use of analgesic/sedative medications, ICU LOS, and duration of MV.

According the results of two RCT study (Level of evidence $1+)^{[40,41]}$, one before and after prospective study (Level of evidence $2++)^{[42]}$, and the recommendations of one analgesia/sedation guidelines ${ }^{[39]}$, it was concluded to use Behavioral Pain Scale (BPS) or the Critical-Care Pain Observation Tool (CPOT) for pain monitoring (Recommendation number 1.2 Box 6). These studies revealed that BPS and CPOT are the most valid and reliable behavioral pain scales for pain monitoring in medical, postoperative, or trauma adult ICU patients. Their regular use can lead to better pain management and improved clinical outcomes in ICU patients. In addition two descriptive studies (Level of evidence $1+)^{[30,43]}$ provide consistent evidence that the BPS and CPOT scales have good psychometric properties in terms of inter-rater reliability and discriminant validity in ICU patients.

According to three descriptive studies (Level of evidence $2++)^{[30,43,44]}$, and one analgesia/sedation guidelines $^{[39]}$, it was recommended that vital signs should not be used alone for pain assessment in adult ICU patients. Vital signs may be used as a cue to begin further pain assessment (Recommendation number 1.3 Box 6). These descriptive studies provide inconsistent evidence of the validity of vital signs for the purpose of pain assessment in medical, postoperative, and trauma ICU patients. Even if there is a trend for vital signs to increase when critically ill patients are exposed to painful procedures, these increases are not reliable predictors of pain.

\subsubsection{Pain management recommendations:}

Based on one quasi-experimental study (Level of evidence $1+)^{[45]}$ and analgesia/sedation guidelines ${ }^{[39]}$, it was recommended to perform non-pharmacologic interventions and/or administer pre-procedural analgesia to alleviate pain in adult ICU patients' prior invasive and potentially painful procedures (Recommendation number 2.1 Box 6). Significantly lower pain scores were reported by patients if they received relaxation plus analgesics prior to invasive procedures. ${ }^{[45]}$

On the basis of one RCT study (Level of evidence1+) ${ }^{[25]}$ and one sedation and analgesia guidelines ${ }^{[46]}$, fentanyl should be given for acutely distressed patients with hemodynamic instability or renal insufficiency. (Recommendation number 2.2 Box 6). Fentanyl is the drug of choice for a rapid onset of analgesia in acutely distressed patients due to its relative lack of histamine release and greater hemodynamic stability. It has a rapid onset of action and a short duration of action from redistribution. ${ }^{[46]}$ 
According to one RCT study (Level of evidence1+) ${ }^{[40]}$ two prospective cohort study (Level of evidence $2++)^{[37,42]}$, one retrospective cohort study (Level of evidence $\left.2++\right)^{[47]}$ and one sedation and analgesia guidelines ${ }^{[46]}$, morphine should be given for hemodynamically stable patients (Recommendation number 2.3 Box 6). These studies documented that morphine is a rapid-acting agent that can treat the acute distressed patients. Moreover, it lasts relatively longer so it can enhance the analgesic effect. Its use has been associated with histamine-related hemodynamic change and impaired clearance in patients with renal failure.

\subsubsection{Sedation/agitation assessment recommendations:}

On the basis of three RCT studies (Level of evidence $1+)^{[8,25,40]}$, one prospective cohort study (Level of evidence $2++)^{[42]}$, one retrospective cohort study (Level of evidence $\left.2++\right)^{[47]}$ and two guidelines ${ }^{[39,46]}$, patient's sedation level should be assess regularly (Recommendation number 3.1 Box 6). Because frequently assessing the degree of sedation or agitation may facilitate the management of sedation and so promote patient comfort and safety.

According to one inter-reliability study (Level of evidence $2++)^{[48]}$, one prospective cohort study (Level of evidence $2++)^{[49]}$ and one pain and sedation guidelines ${ }^{[39]}$ it was recommended to use Richmond AgitationSedation Scale (RASS) or Sedation-Agitation Scale (SAS) for sedation assessment. The results of these studies revealed that RASS and SAS are the most valid and reliable sedation assessment tools for use in critically ill patients. Both scales demonstrated a high degree of inter-rater reliability, which included ICU clinicians. Also, both scales were able to discriminate different sedation levels in various clinical situations. (Recommendation number 3.2 Box 6).

\subsubsection{Sedation/agitation management recommendations:}

On the basis of one RCT (Level of evidence $1+)^{[40]}$ one prospective cohort study (Level of evidence:2++) ${ }^{[42]}$ and one retrospective cohort study (Level of evidence $2++)^{[47]}$, start sedation of agitated mechanically ventilated patients only AFTER providing adequate analgesic and managing reversible physical causes (Recommendation number 4.1 Box 6). These studies reported that patients' agitation can be caused by unrelieved pain and discomfort, which could be induced by the monitoring and therapeutic devices (such as ventilator, endotracheal tube and drains).

According to four prospective cohort study (Level of evidence $2++)^{[8,36,37,42]}$ and three RCT studies (Level of evidence $1+)^{[24,40,41]}$, sedation goal should be established to keep patient purposely follows commands without agitation (RASS -2-0) rather than a deep level of sedation (Recommendation number 4.2 Box 6). They reported that promoting patients' comfort and cooperation can facilitate the synchronization and weaning of ventilator. Also, maintaining light levels of sedation in adult ICU patients is associated with improved clinical outcomes (e.g., shorter duration of MV and a shorter ICU LOS). The two RCT studies demonstrated that deeper sedation levels are associated with longer durations of MV and ICU LOS. ${ }^{[24,41]}$

On the basis of two prospective study (Level of evidence $2++)^{[8,42]}$ and one retrospective study (Level of evidence $2++)^{[47]}$, analgesic and sedative drugs selection should be based upon patient needs, drug allergies, and/or organ dysfunction (particularly renal or hepatic dysfunction) because sedations may influence the patients' vital signs (Recommendation number 4.3 Box 6).

According to two RCT studies (Level of evidence $1+)^{[25,41]}$, one prospective study (Level of evidence $2++)^{[42]}$ and one retrospective study (Level of evidence $\left.2++\right)^{[47]}$, midazolam recommended to be used for rapid sedation of acutely agitated patients and for short-term use only (Recommendation number 4.4 Box 6). These studies reported that midazolam has rapid onset time; it provides a fast sedative effect for agitated patients. However it is a long-acting metabolite. The accumulation and prolonged duration of sedative effects could produce unpredictable awakening and time to extubation when infusion continues longer then 48-72 hours.

Based on the results of two RCT studies (Level of evidence $1+)^{[25,41]}$, one prospective study (Level of evidence $2++)^{[42]}$ and recommendations of two guidelines ${ }^{[39,46]}$, propofol recommended to be used for intravenous sedation infusion (Recommendation number 4.5 Box 6). They stated that receiving propofol had statistically more predictable rapid awakening time, which was associated with a reduction in the duration of MV and length of ICU stay.

\subsubsection{General recommendations for both pain and agitation}

Sedation protocol/algorithm should be routinely used in adult ICU patients (Recommendation number 5.1 Box 6). Sedation management by protocol/algorithm has been studied and is clearly advantageous. In RCT study (Level of evidence $1+)^{[24]}$, sedation management of 321 mechanically ventilated patients according to a nurse implemented protocol was associated with significantly shorter duration of $\mathrm{MV}$, decreased rate of tracheostomy, and improved ICU and hospital LOS. Also, one RCT study (Level of evidence 1+) ${ }^{[41]}$ and two prospective cohort studies (Level of evidence $2++)^{[8,42]}$ which examined the introduction of a sedation protocol and algorithm on the duration of MV using before-after design. Most of studies observed a significant reduction 
in the average duration of MV in the protocol/or algorithm group.

It is recommended to use non-pharmacological interventions to promote sleep and increase overall patient comfort (Recommendation number 5.2 Box 6). According to Jacobi guidelines (2002) ${ }^{[6]}$, implementing quiet time on both day and night shifts and clustering patient care activities reduce disturbances and promote sleep in adult ICU patients. Relaxation will lead to a parasympathetic response and a decrease in respiratory rate, heart rate, jaw tension, and blood pressure. Also, music can decrease heart rate, respiratory rate, myocardial oxygen demand, and anxiety scores and improve sleep. In addition, back massage is an alternative or adjunct to pharmacologic therapy in critically ill patients. ${ }^{[6]}$

\subsection{Part III: Analgo-sedation guidelines appraisal}

Table 7 presents results of appraisers' evaluation of analgo-sedation guidelines quality according to domains' scores of AGREE II instrument. Very high scores were noted for Scope and Purpose, Stakeholder Involvement and Applicability $(87.3 \%, 82.6 \%$ and 81.5$)$ respectively. By contrast, the lowest domain scores were obtained by Clarity and Presentation and Editorial Independence (78.5\% and 78.8\%) respectively.

The domain Scope and Purpose is concerned with the overall aim of the guidelines, specific health questions and whether the target population is specifically described. The mean score of this domain was $87.3 \%$. Most of appraisers reported that this domain was very precise in describing the aims, health questions and populations. The mean score of the domain Stakeholder Involvement was $82.6 \%$. Most of appraisers mentioned that the involvement of appropriate stakeholders was best described in the current guidelines. The domain Applicability describes how the guidelines implementation is facilitated. This domain had relatively high score $(81.5 \%)$. The current guidelines were supported with application tools as (The Critical-Care Pain Observation Tool (CPOT), Richmond Agitation Sedation Scale (RASS), analgo-sedation algorithm, and ICU analgesia and sedation order sheet). All appraisers welcomed these supportive tools and recommended its application in clinical practice. Regarding Clarity and Presentation, the appraisers differed in their opinion, mainly on easy identification of the key recommendations. The guidelines had the lowest score in this domain (78.5\%). To improve the current guidelines' clarity and presentation quality, some appraisers suggested that presenting the guidelines in two versions would be of benefit: one containing a detailed description of methodology and evidence, and a simpler version with key messages only. This would allow target users to capture the key recommendations easily and improving compliance with best practice.

The domain Editorial Independence assesses whether the final recommendations were influenced by the views or interests of a funding body or whether competing interests of the guidelines authors were addressed. Some appraisers mentioned that transparency of opinion was not illustrated in the current guidelines.

Table 8 illustrates appraisers' opinion of the overall quality of the developed analgo-sedation guidelines. The appraisers evaluated the overall quality of analgo-sedation guidelines using numeric scores (higher scores suggest a higher quality). The current guidelines had received scores in the higher end of the spectrum of quality among most of appraisers.

Table 9 shows appraisers' recommendations for the use of analgo-sedation guidelines. The majority (71.4\%) of academic nursing staff as well as two thirds $(66.6 \%)$ of academic medical staff recommended the current guidelines to be used without modifications. Also, all practitioners (nurses and physicians) recommended it without modifications.

\section{Discussion}

Properly used pain and sedation protocols/guidelines in the ICU, which are primarily driven by nurses, is one of the main cornerstones in the treatment of the mechanically ventilated patients. ${ }^{[1,11]}$ To accomplish the development of the intended evidence based analgo-sedation guidelines in the present study, it was necessary to explore how routine analgo-sedation practices provided by CCNs in ICU setting influenced patient's clinical outcomes. Despite the use of written sedation guidelines is strongly encouraged as a way to promote a consistent approach in sedation practices ${ }^{[1,11]}$, it was observed that CCNs and ICU physicians in the current study did not have written sedation protocol/guidelines or sedation/pain assessment tools. Consequently these affected the patients' clinical outcomes in the intended study setting.

During the observational phase of the current study, it was found that the most common opioid to promote patient comfort was fentanyl. While propofol, midazolam were the agents most commonly used for sedation. The results of the current study are consistent with other surveys that identified these drugs as the top of choices for both short-term and prolonged sedation. ${ }^{[9,19,20]}$ There was no recorded use of morphine during the current study, suggesting that this opioid is not widely used in the current study setting. In contrast, an Australian-New Zealand survey performed in 2010 showed that morphine was used as the first choice for analgesia followed by fentanyl. ${ }^{[50]}$ These findings may be indicative of a cultural difference regarding the approach to analgesia. 
The management of analgesia/sedation therapy often differs among physicians in the current ICU setting. The choice of certain sedatives or analgesics in the present study most likely were influenced by many factors, including the severity of patient's illness, the foreseeable duration of MV, physicians' preferences, and the habits and resources of each site, as shown previously. ${ }^{[2,19,20]}$ Our findings indicate also that sedative/analgesic regimens were not adjusted probably because studied patients were not evaluated by CCNs to determine whether changes in drug dosages were necessary to maintain comfort and sedation level. Contrary, in Singapore Koh et al. $(2010)^{[51]}$ found that the majority $(84.2 \%)$ of the mechanically ventilated patients who were sedated was monitored with sedation scales either Ramsay or Riker Sedation Scales.

There is strong evidence that intermittent bolus administration of sedatives, daily interruption of sedative infusion, or sedation according to guidelines/protocols is more advantageous than the continuous infusion of sedative drugs. ${ }^{[4]}$ Unfortunately, it was observed during the current study that continuous intravenous infusion was the most frequent route of administrating sedatives and/or analgesics. Also, the practice of daily sedation interruption has not yet been implemented in the studied ICU, creating a large evidence-to-practice gap. In contrast, Koh et al. $(2010)^{[51]}$ reported that most patients $(70.8 \%)$ had daily interruption of sedation.

Although sedation guidelines recommend that sedation be started only after providing adequate analgesia ${ }^{[6,39]}$, the patients in the present study did not receive analgesics or pain management as needed and was sometimes ignored. In addition, the titration of sedatives/analgesics in the current study was done by the ICU physicians. However, a de Wit et al. study $(2008)^{[25]}$ had shown that a nurse-implemented sedation protocol had resulted in improved outcomes with faster resolution of critical illness and shorter ICU and hospital stay.

The major findings of the observational phase of the current study include that the studied patients were kept in deep states of sedation most of the study period. There are several possible explanations about this. First, CCNs in the intended ICU usually preferred to have patients sedated, because this sedated state is mistakenly believed to facilitate procedures, nursing care and prevent accidental extubation. Second, there are neither validated instruments to objectively measure patient's sedation levels nor a standardized sedation protocols/guidelines being used in the study setting. This is supported by another Egyptian study done by Ali $(2015)^{[34]}$ who documented that more than half of the CCNs had low level of sedation practices. The researcher found that all CCNs did not use sedation protocols/guidelines or sedation scales during their sedation practices. Also, in a survey of 423 members of the American Association of Critical Care Nurses, nearly half of respondents indicated their intention to sedate all of their mechanically ventilated patients. ${ }^{[52]}$ These findings suggest a widespread culture of keeping mechanically ventilated ICU patients at deep levels of sedation in order to facilitate ICU patient care activities. The results of the current study are in agreement with Payen et al. $(2007)^{[2]}$ who found that $41 \%$ to $57 \%$ of assessed patients were in a deep sedation state and Grap et al. $(2012)^{[53]}$ who observed that patients spent $80 \%$ of the time in mild/deep sedation and only $20 \%$ awake/alert.

It was found during the present study that all patients experienced pain all over the study period and the pain score was remained within moderate level using CPOT. This in agreement with the study of Al Sutari et al. $(2014)^{[54]}$ which revealed that mechanically ventilated patients experience pain during rest as well as during routine nursing interventions. In the contrary, findings of a study conducted by Elliott et al., (2013) ${ }^{[55]}$ who reported that over half the patients had no pain. It was observed during the current study that all nurses didn't use any clinically-validated pain assessment tool. The possible explanation of this that routine pain assessment may be believed by CCNs as a time-consuming process and have no visible impact on patients' outcome. This may weaken their motivation in the use of instruments to measure analgesia as a standard of practice. Also, the researchers interpret this practice due to increased nurses' work load and lack of education, supervision and cooperation between multidisciplinary health team members (nurse-physician). This supported by the results of Badr et al. study $(2015)^{[56]}$ which conducted at the ICUs of Cairo University Hospitals. They found that the majority of the CCNs (95\%) had got unsatisfactory level of pain assessment practices. In the contrary, Payen et al. $(2007)^{[2]}$ found that $40 \%$ of studied patient sample was assessed by CCNs for pain. These differences in the pain practices may be attributed to differences in ongoing professional education received and presence of inconsistent guidelines among nurses.

During the present study there were controversial findings regarding the occurrences of adverse events including unintentional removal of ETT, urinary catheter, peripheral venous line, naso-gastric tube, central venous catheter and/or drainage tubes. The occurrences of these adverse events were high, although the studied patients were in states of sedation in the most of the study time. This can be explained by that these adverse events were detected at the time were the patients in state of agitation, as it was found that more than half of the studied patients were in a combative state (RASS score +1 to +4 ) during the first 48 hours of ICU admission. Similarly studies reported rates of self-extubation. ${ }^{[8,36,41]}$ More than quarter of the studied patients were diagnosed as having ventilator-associated pneumonia; this may be due to absence of sedation assessment. This point of view is consistent with Payen et al. $2007^{[2]}$ who reported that systematic assessments of pain and sedation could possibly have impact on patients' outcome by reducing the duration of $\mathrm{MV}$ and the rate of nosocomial infections. Moreover during the present study, more half of patients were being restrained; all had 
wrist, arm or ankle restraints. Similarly, Koh et al. $(2010)^{[51]}$ found that one third of patients being physically restrained.

The results of this observational phase offer important insights into practices for sedation and analgesia in mechanically ventilated patients in Egypt. This study reflects what is really done by CCNs in intended ICU, provided data about patients' clinical outcomes of routine sedation and analgesia practices, pointed out gaps between clinical practice and current recommendations, and served as a basis for the elaboration of intended analgo-sedation guidelines.

The findings of the observational phase of the current study are crucial and warrant a change in the way sedation/pain assessment and management is implemented in adult ICUs in Egypt. In response to these findings, it was mandatory to the authors to develop evidence based analgo-sedation guidelines that will assist CCNs to intervene consistently with adult mechanically ventilated patients.

Over the past decade, a significant number of sedation guidelines and protocols have been written and published but these guidelines were from the physicians' prospective $\mathrm{e}^{[6,23,39,46]}$. Overall, almost guidelines were performed poorly with respect to the nursing role. The analgesia and sedation guidelines published in 2002 by task force of the American College of Critical Care Medicine, American Society of Health-System Pharmacists, and American College of Chest Physicians ${ }^{[6]}$; guidelines for sedation and analgesia by Shapiro et al. in 2007 ${ }^{[46]}$ and guidelines for the management of pain, agitation, and delirium in $2013^{[39]}$ provided strong foundation for the present work. However, these documents did not explicitly outline how evidence was identified, interpreted, or integrated into recommendations. Guidelines of Shapiro et al. in $2007^{[46]}$ did not provide sufficient methodology describing how the guidelines were developed, especially for the searches of electronic database and rating scheme for the strength of evidence. Although these guidelines mentioned that nurses are one of their intended users, it did not directly mention what they should do, while the present guidelines identified the role of nurse clearly and directly.

The challenging and controversial nature of the subjective assessment of sedation level and requirements has probably contributed to uncertainty in developing clinical practice sedation guidelines for ICU patients. Therefore only a few guidelines exist to guide the clinician in administering sedation to ICU patients. ${ }^{[1]}$ Nevertheless the use of simple guidelines based on a target sedation level has led to some impressive improvements in outcomes. ${ }^{[24,36,37]}$ Benefits reported include reduced duration of ventilation, reduced length of stay in ICU and reduced costs. Bratteb $\varnothing$, et al. (2004) introduced simple guidelines for the management of sedation in adult mechanically ventilated patients. Implementation strategies used in this study were successful in encouraging clinicians to use guidelines and in achieving demonstrable improvements in patient outcomes. The mean ventilator time was reduced by nearly $30 \% .^{[36]}$

The current analgo-sedation guidelines demonstrate several strength points. Firstly, it developed by multidisciplinary panel and the panel's balance of critical care nursing, critical care medicine, university-based and clinicians. Second, the present guidelines were based on systematic review of the scientific evidence and different types of studies were included (RCTs, retrospective and prospective cohort studies, case-control, cross sectional studies and clinical guidelines). Third, guidelines' recommendations are explicitly linked to the supporting evidence and graded according to the strength of that evidence. Fourth, the current guidelines are attached with supportive tools (analgo-sedation algorithm, pain/sedation assessment tools and ICU analgesia/sedation order sheet) which will facilitate the nursing interventions. Finally, these guidelines provided a frame work for nursing decision making and patient assessment during analgesia/sedation practices.

The analgo-sedation algorithm developed during the current study is useful for busy CCNs because it is easily interpreted and does not require the user to read large amounts of text to make a clinical decision. Sedation algorithm previously studied by Brook, et al. (1999) in a North American medical ICU. By using this algorithm nurses were able to determine the type and dose of sedative and the need for continuous infusion based on sedation level assessment. The nurses were able to initiate the medication without a prescription from the medical officer. ${ }^{[24]}$

Other strengths of the present guidelines include the process of its development which followed quality criteria for developing guidelines produced by Scottish Intercollegiate Guidelines Network (SIGN) ${ }^{[32]}$ and National Institute for Health and Clinical Excellence (NICE). ${ }^{[57]}$ These sources provided greater details on the methodology of guidelines development. These principles will remain basic to the development of valid and usable guidelines which include proper selection of guidelines' topic, form of multidisciplinary group of work, developing clinical questions, comprehensively review of literature, rating of articles, and summarizing finding. Moreover, the successful guidelines should include recommendations reviewed and critically appraised by a group of expert reviewers and intended users by using a standardized tool.

The current guidelines were evaluated by external appraisers to ensure its content validity, clarity and applicability. The advantages of using a group to evaluate the guidelines include sharing of work among group members, reduced potential for bias in the evaluation process and increased awareness of guidelines. The appraisers' evaluation of the present guidelines was based on the application of AGREE II instrument. ${ }^{[28]}$ This 
tool is one of the few guidelines assessment tools to demonstrate validity and reliability. Furthermore, the areas covered by the AGREE II instrument are logical for anyone to consider when conducting guidelines development or evaluation. The present study showed that the guidelines quality can be relatively reliably assessed with the AGREE II instrument, as seen from its use in other study characterized by high reliability. ${ }^{[58]}$ The AGREE II instrument evaluates the quality of guidelines by assessing the development methods and related properties. It is mainly concerned with how quality is maintained during the process of guidelines development. ${ }^{[28,58]}$ Although some might want to view the results from this quantitative evaluation (AGREE II instrument) as an objective measure of guidelines' quality, it is important to remember that scores are influenced by the extent to which the guidelines developers described the methods used to develop the guidelines. Rigorously developed guidelines may score poorly if the process was not well described.

\section{Limitations of the study}

Our study has limitations that should be noted. First, we did not directly measure the level of nursing compliance with the analgo-sedation guidelines. Therefore, we cannot estimate whether additional benefits could be gained by further adherence to the analgo-sedation guidelines. Second, we did not assess the impact of analgo-sedation guidelines on patients' clinical outcomes that could influence its clinical application.

\section{Conclusion}

Based on the findings of the present study, it can be concluded that CCNs in the intended ICU setting did not have written sedation protocol/guidelines or sedation/pain assessment tools. Unsatisfactory nursing practices were found during the observational phase of the current study regarding sedation/analgesia practices which had negative effects on patients' clinical outcomes. The studied patients were kept in deep states of sedation most of the study period. All studied patients experienced pain all over the study period. More than half of studied patients removed their ETT at least once during their stay. About $47.5 \%$ and $40 \%$ of them had an accidental removal of urinary catheter and peripheral venous line respectively. Physical restraint was performed in about $55 \%$ of studied sample. Half of the studied patients were subjected to weaning failure and $32.5 \%$ of them diagnosed as ventilator-associated pneumonia. The observational phase findings provide additional rationale for the application of standardized approach in sedation management for mechanically ventilated patients. In response to this, the authors developed evidence based analgo-sedation guidelines. The overall quality of guidelines assessed in the present study was relatively high, particularly in the domains Scope and Purpose, Stakeholder Involvement and Applicability. By contrast, lowest quality was found in the domains Clarity and Presentation. The majority of academic nursing appraisers as well as two thirds of academic medical appraisers recommended the current analgo-sedation guidelines without modifications. All practitioner appraisers also recommended it without modifications. Most of appraisers mentioned that the current guidelines are effective and could be easily implemented by nurses and they are in need for such guidelines. Furthermore, they welcomed the guidelines as a tool for evaluation of nursing performance.

\section{Recommendations}

On the basis of the most important findings of the study, the following recommendations are suggested:

- Implementation of analgo-sedation guidelines in clinical practice for adult mechanically ventilated patients is highly recommended.

- Pain and sedation assessment using the validated scales (CPOT \& RASS) should be conducted by the CCNs.

- Collaborative care planning that improve communication among critical care members should be applied while dealing with patients requiring sedation for $\geq 48$ hours using the developed sedation/analgesia order sheet and analgo-sedation algorithm.

- The critical care managers should establish unit policy to incorporate analgo-sedation guidelines in clinical practice as a routine of unit care.

- It is strongly recommended for CCNs to attain educational programs about application of analgo-sedation guidelines in their clinical area.

- Development of an audit and feedback mechanism to monitor adherence to the intended guidelines and determine whether the guidelines has impacted patient clinical outcomes need to be studied further.

- Studies are needed to test cost effectiveness of implementation of analgo-sedation guidelines.

- The barriers to implementation of analgo-sedation guidelines need to be studied further.

\section{Acknowledgements}


The authors expresses their deepest gratitude and sincerest appreciation to all those who have contributed to the development and revision of these guidelines. Also we deeply appreciate the valuable cooperation of all staff member (residents and nurses) in the ICU of Emergency Hospital at Mansoura University.

\section{Conflicts of interest disclosure}

The authors declare that there is no conflict of interest statement.

\section{References}

[1]. Ki W. Development and evaluation of a sedation protocol of weaning off the mechanical ventilation. Master thesis; University of Hong Kong; 2011.

[2]. Payen, J.F., Chanques, G., Mantz, J., Hercule, C., Auriant, I., Leguillou, J.L., Binhas, M., Genty, C., Rolland, C., and Bosson, J.L. Current practices in sedation and analgesia for mechanically ventilated critically ill patients: A prospective multicenter patientbased study. Anesthesiology. 2007; 106(4):687-95. doi:10.1097/01.anes.0000264747.09017.da.

[3]. Tate, J., Dabbs, A., Hoffman, L., Milbrandt, E., and Happ, M. Anxiety and Agitation in Mechanically Ventilated Patients. Qual Health Res. 2012; 22(2): 157-73. doi:10.1177/1049732311421616.

[4]. Chlan, L.L. Description of anxiety levels by individual differences and clinical factors in patients receiving mechanical ventilatory support. Heart \& Lung 2003;32(4):275-82.

[5]. Mehta, S., McCullagh, I., and Burry, L. Current sedation practices: lessons learned from international surveys. Critical Care Clinics. $2009 ; 25(3): 471-88$

[6]. Jacobi, J., Fraser G.L., Coursin, D.B., et al; Task Force of the American College of Critical Care Medicine (ACCM) of the Society of Critical Care Medicine (SCCM), American Society of Health-System Pharmacists (ASHP), American College of Chest Physicians. Clinical practice guidelines for the sustained use of sedatives and analgesics in the critically ill adult. Crit Care Med. 2002; 30(1):119-41.

[7]. Iakovou, A., Lama, K., and Tsegaye, A. Update on Sedation in the Critical Care Unit. The Open Critical Care Medicine Journal 2013; 6 (Suppl 1: M5):66-79.

[8]. Quenot, J.P., Ladoire, S., Devoucoux, F,. Doise, J.M., Cailliod, R., Cunin, N., Aubé, H., Blettery, B., and Charles, P.E. Effect of a nurse-implemented sedation protocol on the incidence of ventilator-associated pneumonia. Crit Care Med. 2007; 35(9):2031-6.

[9]. Wøien, H. The significance of a systematic approach in intensive care pain treatment and sedation: A descriptive and explorative study of nurses' and physicians' practice in the assessment of mechanically ventilated intensive care patients' analgesic and sedative needs. Doctoral thesis; Institute of Health and Society; Department of Nursing Science University of Oslo, Norway; 2012. http://urn.nb.no/URN:NBN:no-34393.

[10]. Keogh, S., Long, D., and Horn, D. Practice guidelines for sedation and analgesia management of critically ill children: a pilot study evaluating guideline impact and feasibility in the PICU. BMJ Open. 2015; 5:e006428. doi:10.1136/bmjopen-2014-006428.

[11]. Elliott, R. The effect of an algorithm based sedation guideline on the duration of mechanical ventilation for intensive care patients in an Australian intensive care unit. Master thesis, Faculty of Nursing, Midwifery and Health, University of Technology, Sydney 2005.

[12]. Aitken, L., Bucknall, T., Kent, B., Mitchell, M., Burmeister, E., and Keogh, S. Sedation protocols to reduce duration of mechanical ventilation in the ICU: a Cochrane Systematic Review. Journal of Advanced Nursing. 2016;72(2):261-72. doi: 10.1111/jan.12843.

[13]. Schweickert, W.D., Gehlbach, B.K., Pohlman, A.S., Hall, J.B., and Kress, J.P. Daily interruption of sedative infusions and complications of critical illness in mechanically ventilated patients. Crit Care Med. 2004; 32(6):1272-6.

[14]. Walker, N., and Gillen, P. Investigating nurses' perceptions of their role in managing sedation in intensive care: An exploratory study. Intensive and Critical Care Nursing. 2006; 22(6):338-45.

[15]. Yilmaz, C., Girgin, N., Ozdemir, N., and Kutlay, O. The effect of nursing-implemented sedation on the duration of mechanical ventilation in the ICU. Ulus Travma Acil Cerrahi Derg. 2010;16(6):521-6.

[16]. Lawner, B., Farzad, A., Keegan, S., and Knight W. Sedation of the mechanically ventilated patient in the emergency department. EM Critical Care. 2014; 4(2):1-16.

[17]. Sessler, C.N., and Pedram, S. Protocolized and target-based sedation and analgesia in the ICU. Critical Care Clinics. 2009; 25(3):489-513. doi:10.1016/j.ccc.2009.03.001.

[18]. Wøien, H., Stubhaug, A., and Bjørk, I. Analgesia and sedation of mechanically ventilated patients-a national survey of clinical practice. Acta Anaesthesiol Scand. 2012; 56: 23-9. DOI: 10.1111/j.1399-6576.2011.02524.x.

[19]. Egerod, I., Christensen, B.V., and Johansen, L. Nurses' and physicians' sedation practices in Danish ICUs in 2003 A national survey. Intensive Crit Care Nurs. 2006; 22(1): 22-31.

[20]. Samuelson, K,A., Larsson, S., Lundberg, D., and Fridlund, B. Intensive care sedation of mechanically ventilated patients: a national Swedish survey. Intensive Crit Care Nurs. 2003; 19: 350-62.

[21]. Mehta, S., Meade, M.O., Hynes, P., Filate, W.A., Burry, L., Hallett, D., McDonald, E., and Cook, D.J. A multicenter survey of Ontario intensive care unit nurses regarding the use of sedatives and analgesics for adults receiving mechanical ventilation. J Crit Care. 2007; 22(3): 191-6.

[22]. Shinotsuka, C., and Salluh, J. Perceptions and practices regarding delirium, sedation and analgesia in critically ill patients: A narrative review. Rev Bras Ter Intensiva. 2013; 25(2):155-61. DOI:10.5935/0103-507X.20130027.

[23]. Hammond, J. Protocols and guidelines in critical care: development and implementation. Current opinion in critical care. 2001; 7(6):464-8.

[24]. Brook, A., Ahrens, T., Schaiff, R., Prentice, D., Sherman, G., Shannon, W., and Kollef, M. Effect of a nursing-implemented sedation protocol on the duration of mechanical ventilation. Crit Care Med. 1999; 27(12):2609-15.

[25]. de Wit, M., Gennings, C., Jenvey, W., and Epstein, S. Randomized trial comparing daily interruption of sedation and nursingimplemented sedation algorithm in medical intensive care unit patients. Critical Care. 2008; 12(3):R70. doi:10.1186/cc6908. Available online http://ccforum.com/content/12/3/R70.

[26]. Sessler, C.N., Gosnell, M.S., Grap, M.J., Brophy, G.M, O'Neal, P.V, Keane, K.A, Tesoro, E.P, and Elswick, R.K. The Richmond agitation-sedation scale: validity and reliability in adult intensive care unit patients. Am J Respir Crit Care Med. 2002; 166:1338-44.

[27]. Gelinas, C., Fillion, L., and Puntillo, K. Item selection and content validity of the Critical-Care Pain Observation Tool for nonverbal adults. J Adv Nurs. 2008; 65(1):203-16.

[28]. AGREE Next Steps Research Consortium. AGREE II: advancing guideline development, reporting and evaluation in healthcare. http://www.agreetrust.org; 2010. (Accessed June 30, 2015).

[29]. Mansouri, P., Javadpour, S., Zand, F., Ghodsbin, F., Sabetian, G., Masjedi, M., and Tabatabaee, H.R.. Implementation of a protocol for integrated management of pain, agitation, and delirium can improve clinical outcomes in the intensive care unit: A randomized clinical trial. Journal of Critical Care 2013; 28:918-22. 
[30]. Gelinas, C., and Johnston, C. Pain assessment in the critically ill ventilated adult: validation of the Critical-Care Observational Tool and physiologic indicators. Clin J Pain. 2007; 23(6):497-505.

[31]. MacDermid, J.C, Brooks, D., Solway, S., Switzer-McIntyre, S., Brosseau, L., and Graham, I.D. Reliability and validity of the AGREE instrument used by physical therapists in assessment of clinical practice guidelines. BMC Health Serv Res. 2005; 5:18. Available: http://www.ncbi.nlm.nih.gov/pmc/articles/PMC555572/?tool = pubmed. (Accessed: 2015 February 9).

[32]. Scottish Intercollegiate Guidelines Network. SIGN Guideline Development Handbook: SIGN 50. (2008). Retrieved 1 August, 2008 from http://www.sign.ac.uk/guidelines/fulltext/50/index.html.

[33]. El-Bakour, A. Critical care nurses' knowledge and practice regarding the safe use of sedatives. Master thesis, Alexandria University, Faculty of Nursing, Egypt, 2012.

[34]. Ali, B. Factors affecting critical care nurses practices related to sedation administration in the critical care units. Master thesis, Alexandria University, Faculty of Nursing, Egypt, 2015.

[35]. Glasziou, P., Del Mar, C., and Salisbury, J. Evidence-based practice workbook: bridging the gap between health care research and practice. $2^{\text {nd }}$ ed. Oxford, UK: Blackwell Publishing Ltd.; 2007. 445-56.

[36]. Brattebo, G., Hofoss, D., Flaatten, H., Muri, A., Gjerde, S., and Plsek, P. Effect of a scoring system and protocol for sedation on duration of patients' need for ventilator support in a surgical intensive care unit. Qual Saf Health Care. 2004; 13(3): 203-5.

[37]. De Jonghe, B., Bastuji-Garin, S., Fangio, P., Lacherade, J.C., Jabot, J., Appéré-De-Vecchi, C., Rocha, N., and Outin H.. Sedation algorithm in critically ill patients without acute brain injury. Crit Care Med. 2005; 33(1):120-7.

[38]. Payen, J.F., Bosson, J.L., Chanques, G., Mantz, J., Labarere, J., and DOLOREA Investigators: Pain assessment is associated with decreased duration of mechanical ventilation in the intensive care unit: a post hoc analysis of the DOLOREA study. Anesthesiology. 2009; 111:1308-16. doi:10.1097/ALN.0b013e3181c0d4f0.

[39]. Barr, J., Fraser, G., Puntillo, K., et al. Clinical practice guidelines for the management of pain, agitation, and delirium in adult patients in the Intensive Care Unit. Critical Care Medicine. 2013; 41(1):264-306. DOI: 10.1097/CCM.0b013e3182783b72.

[40]. Mehta, S., Burry, L., Martinez-Motta, J.C., et al. A randomized trial of daily awakening in critically ill patients managed with a sedation protocol: a pilot trial. Critical Care Medicine. 2008; 36(7): 2092-99.

[41]. Girard, T.D., Kress, J.P., Fuchs, B.D., et al. Efficacy and safety of a paired sedation and ventilator weaning protocol for mechanically ventilated patients in intensive care (Awakening and Breathing Controlled trial): a randomized controlled trial. Lancet. 2008; 371: 126-34.

[42]. Aria-Rivera, S., Sanchez-Sanchez, M.M., Santoz-Diaz, R., Gallardo-Murillo, J., Sánchez-Izquierdo, R., Frutos-Vivar, F., Ferguson, N.D., and Esteban, A. Effect of a nursing implemented sedation protocol on weaning outcome. Critical Care Medicine 2008; 36: 2054-60.

[43]. Arroyo-Novoa, C.M, Figueroa-Ramos, M.I, Puntillo, K.A., Stanik-Hutt, J., Thompson, C.L., White, C., and Wild, L.R. Pain related to tracheal suctioning in awake acutely and critically ill adults: A descriptive study. Intensive Crit Care Nurs. 2008; 24(1):20-7.

[44]. Gélinas, C., and Arbour, C. Behavioral and physiologic indicators during a nociceptive procedure in conscious and unconscious mechanically ventilated adults: Similar or different? Journal of critical care. 2009; 24(4):628.e7-17. DOI: 10.1016/j.jcrc.2009.01.013.

[45]. Friesner, S.A., Curry, D.M., and Moddeman, G.R. Comparison of two pain-management strategies during chest tube removal: Relaxation exercise with opioids and opioids alone. Heart Lung. 2006; 35:269-76.

[46]. Shapiro, M., West, M., Nathens, A., et al. Guidelines for sedation and analgesia during mechanical ventilation: General Overview. J Trauma. 2007; 63:945-50.

[47]. Jakob, S.M., Lubszky, S., Friolet, R., Rothen, H.U., Kolarova, A., and Takala, J. Sedation and weaning from mechanical ventilation: effects of process optimization outside a clinical trial. Journal of Critical Care. 2007; 22(3):219-28.

[48]. Ryder-Lewis, M.C., and Nelson, K.M. Reliability of the Sedation-Agitation Scale between nurses and doctors. Intensive Crit Care Nurs. 2008; 24:211-17.

[49]. Ely, E.W., Truman, B., Shintani, A., et al. Monitoring sedation status over time in ICU patients: Reliability and validity of the Richmond Agitation-Sedation Scale (RASS). JAMA. 2003; 289(22):2983-91.

[50]. O'Connor, M., Bucknall, T., and Manias, E. Sedation management in Australian and New Zealand intensive care units: doctors' and nurses' practices and opinions. Am J Crit Care. 2010;19(3):285-95.

[51]. Koh ,A., Tee, A., Phoo, J., et al. A national point-prevalence survey of the practice of sedation, analgesia, neuromuscular blockade and delirium assessment in adult intensive care units in Singapore. Crit Care \& Shock. 2010; 13(4):122-31.

[52]. Guttormson, J.L., Chlan, L., Weinert, C., and Savik, K. Factors influencing nurse sedation practices with mechanically ventilated patients: A U.S. national survey. Intensive Crit Care Nurs. 2010; 26(1):44-50. doi: 10.1016/j.iccn.

[53]. Grap, M., Munro, C., Wetzel, P. A., Best, A.M., Ketchum, J.M., Hamilton, V.A., Arief, N.Y., Pickler, R., and Sessler, C.N. Sedation in adults receiving mechanical ventilation: physiological and comfort outcomes. American journal of critical care. 2012 ; 21(3):e54-e63.

[54]. Al Sutari, M., Abdalrahim, M., Hamdan-Mansour, A., and Ayasrah, S. Pain among mechanically ventilated patients in critical care units. J Res Med Sci. 2014; 19(8):726-32.

[55]. Elliott, D., Aitken, L.M., Bucknall, T.K., Seppelt, I.M., Webb, S.A.R., Weisbrodt, L. and McKinley, S. Patient comfort in the intensive care unit: a multicentre, binational point prevalence study of analgesia, sedation and delirium management. Crit Care Resusc. 2013; 15(3): 213-19.

[56]. Badr, M., Morsy, W., and Ali, N. Critical care nurses' knowledge and practices regarding pain assessment and management at Cairo University Hospitals. Egyptian Journal of Nursing. 2015; 10(1): 28-44.

[57]. National Institute for Health and Clinical Excellence (April 2007) the guidelines manual. London: National Institute for Health and Clinical Excellence. Available at www.nice.org.uk

[58]. Jo, M.W., Lee, J.Y., Kim, N.S., Kim, S.Y., Sheen, S., Kim. S.H., and Lee, S.I. Assessment of the quality of clinical practice guidelines in Korea using the AGREE Instrument. Journal of Korean medical science. 2013;28(3): 357-65. 


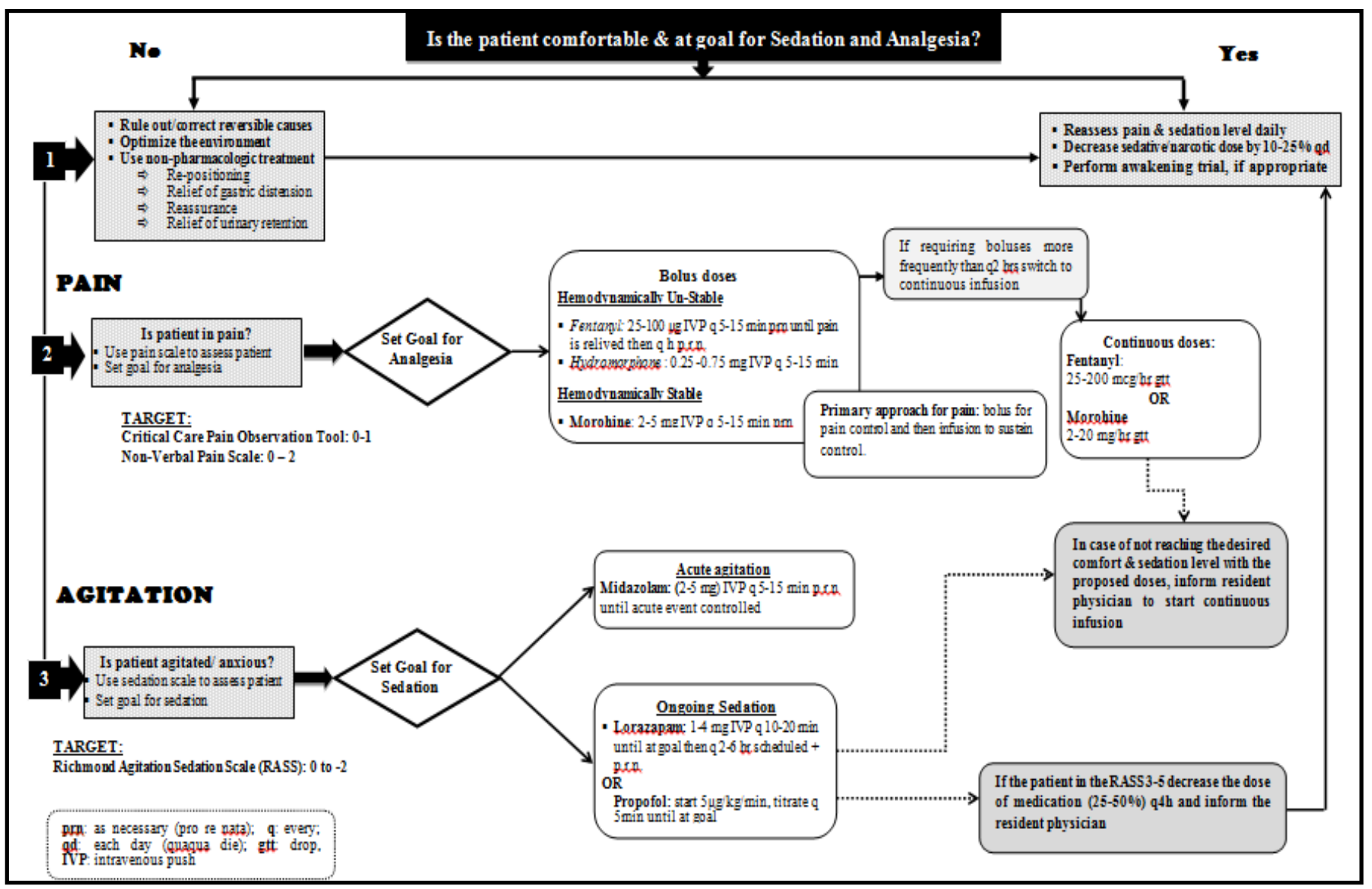

Figure 2. Analgo-sedation algorithm

Table 1 Demographic and clinical characteristics of the studied patients on admission to the intended ICU

\begin{tabular}{|c|c|c|}
\hline Variable & $\mathrm{N}=\mathbf{4 0}$ & $(\%)$ \\
\hline Age (years) $($ mean \pm SD) & \multicolumn{2}{|c|}{$37.1 \pm 1.35$} \\
\hline \multicolumn{3}{|l|}{ Gender } \\
\hline Female & 14 & 35.0 \\
\hline Male & 26 & 65.0 \\
\hline \multicolumn{3}{|l|}{ Type of admission } \\
\hline Medical & 7 & 17.5 \\
\hline Postoperative & 5 & 12.5 \\
\hline Trauma & 28 & 70.0 \\
\hline \multicolumn{3}{|l|}{ Past medical history } \\
\hline No history & 24 & 60.0 \\
\hline Cardiovascular disorders & 4 & 10.0 \\
\hline Respiratory disorders & 6 & 15.0 \\
\hline Neurological disorders & 6 & 15.0 \\
\hline \multicolumn{3}{|l|}{ Reason for mechanical ventilation } \\
\hline COPD exacerbation & 3 & 7.5 \\
\hline Acute respiratory failure & 12 & 30.0 \\
\hline Cardiovascular failure & 2 & 5.0 \\
\hline Neurological disorder & 23 & 57.5 \\
\hline APACHE II on admission (mean \pm SD) & \multicolumn{2}{|c|}{$26.57 \pm 4.9$} \\
\hline GCS on admission (mean \pm SD) & \multicolumn{2}{|c|}{$9.5 \pm 2.2$} \\
\hline
\end{tabular}

APACHE II: Acute Physiology and Chronic Health Evaluation, GCS : Glasgow Coma Scale 
Towards A Standardized Approach For Critical Care Nursing Practices: Development Of Evidence...

Table 2. Patients' Agitation Sedation levels on admission and discharge according to Richmond Agitation Sedation Scale

\begin{tabular}{|c|c|c|c|c|}
\hline \multirow{2}{*}{ RASS } & \multicolumn{2}{|c|}{ On admission } & \multicolumn{2}{|c|}{ On discharge } \\
\hline & $\mathbf{n}$ & $(\%)$ & $\mathbf{n}$ & $(\%)$ \\
\hline Combative $(+1$ to +4$)$ & 22 & 55.0 & 8 & 20 \\
\hline Calm and alert state (0) & 0 & 0.0 & 2 & 5 \\
\hline Sedated $(-1$ to -5$)$ & 18 & 45.0 & 30 & 75 \\
\hline Total RASS $($ Mean \pm SD) & \multicolumn{2}{|l|}{$0.77 \pm 2.7$} & \multicolumn{2}{|c|}{$-0.85 \pm 2.17$} \\
\hline
\end{tabular}

RASS: Richmond Agitation Sedation Scale

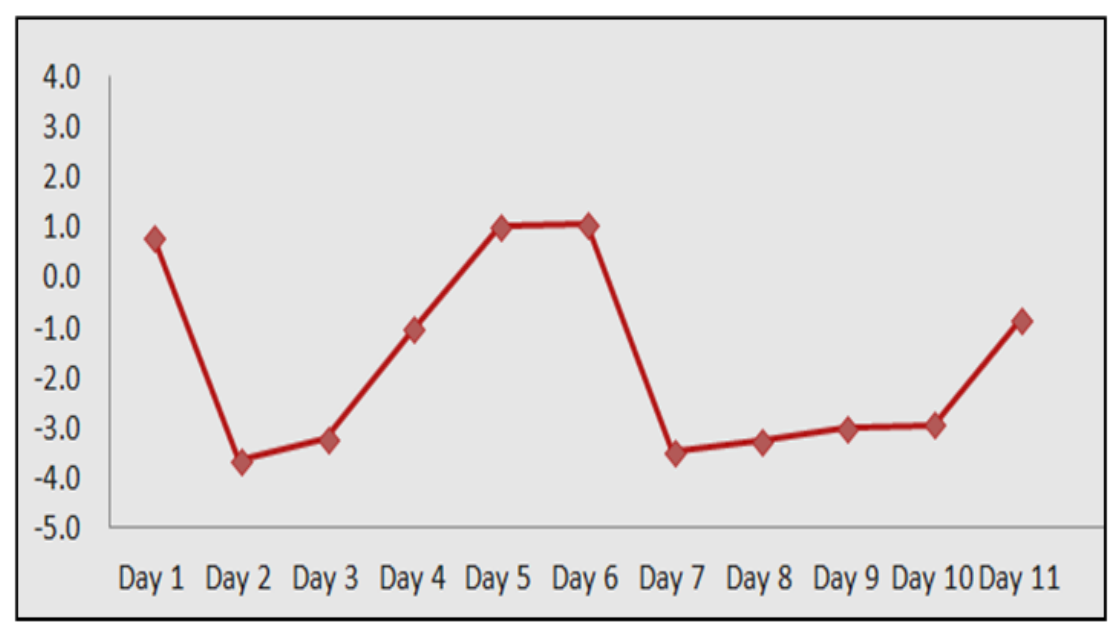

Figure 3 The change of the Richmond Agitation Sedation Score over the study period

Table 3. Number and Percentage of patients regarding pain level using Critical-Care Pain Observation Tool on admission and discharge

\begin{tabular}{|c|c|c|c|c|}
\hline \multirow{2}{*}{ СРОТ } & \multicolumn{2}{|c|}{ On admission } & \multicolumn{2}{|c|}{ On discharge } \\
\hline & $\mathbf{n}$ & $(\%)$ & $\mathbf{n}$ & $(\%)$ \\
\hline No pain (0) & 10 & 25 & 7 & 17.5 \\
\hline Mild pain (1-3) & 1 & 2.5 & 13 & 32.5 \\
\hline Moderate pain (4-5) & 21 & 52.5 & 19 & 47.5 \\
\hline Severe pain (6-8) & 8 & 20.0 & 1 & 2.5 \\
\hline Total CPOT $($ Mean \pm SD) & \multicolumn{2}{|c|}{$3.55 \pm 2.28$} & \multicolumn{2}{|c|}{$3.025 \pm 3.01$} \\
\hline
\end{tabular}

CPOT: Critical-Care Pain Observation Tool

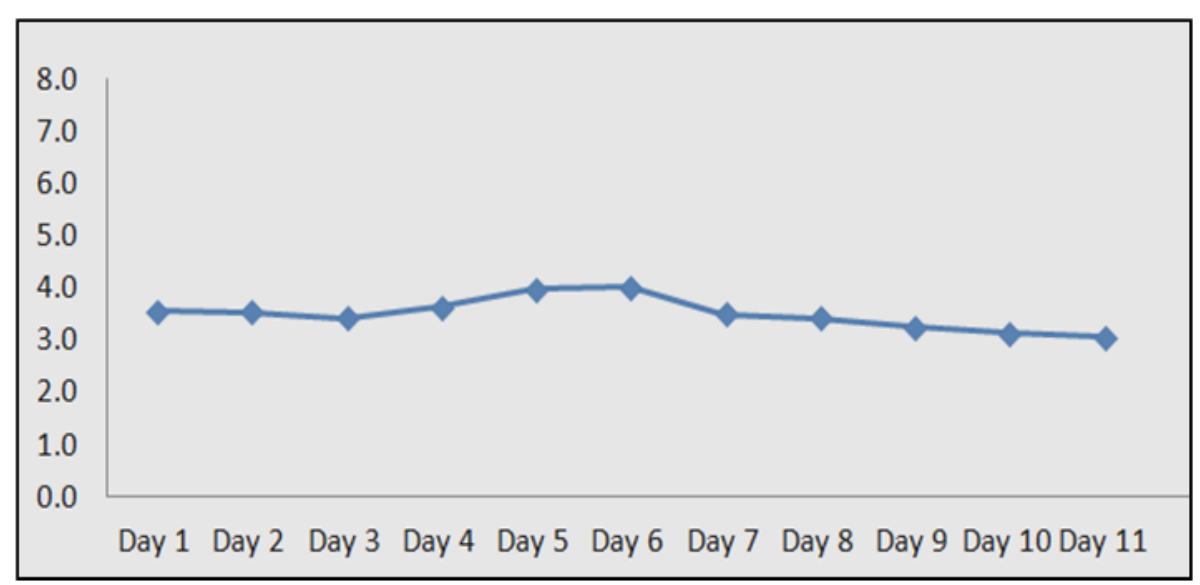

Figure 4 The change of the Critical Care Pain Observation Tool Score over the study period 
Table 4. The mean amounts of drugs used for controlling pain and agitation in the studied sample

\begin{tabular}{|lc|}
\hline \multicolumn{1}{|c|}{ Drug used } & Mean \pm SD dose of drug used \\
\hline Analgesic & \\
Fentanyl $\mathrm{mg} /$ day & $4.12 \pm 6.34$ \\
Sedative & \\
Midazolam mg/day & $520.82 \pm 614.72$ \\
Propofol $\mathrm{mg} /$ day & $2880 \pm 960$ \\
Precedex mcg/day & $1778.82 \pm 5804.1$ \\
\hline
\end{tabular}

Table 5. Occurrence of adverse events throughout the study period in the studied sample

\begin{tabular}{|lcc|}
\hline \multicolumn{1}{|c|}{ Adverse events } & n & $\mathbf{( \% )}$ \\
\hline Unintentional device removal & & \\
$\quad$ Endotracheal tube & 22 & 55.0 \\
Central venous catheter & 8 & 20.0 \\
Naso/oro-gastric tube & 15 & 37.5 \\
Urinary catheter & 19 & 47.5 \\
Drainage tube & 5 & 12.5 \\
Peripheral venous line & 16 & 40.0 \\
Other adverse events & & \\
Reintubation & 22 & 55.0 \\
Tracheostomy & 11 & 27.5 \\
Weaning failure & 20 & 50.0 \\
Ventilator-associated pneumonia & 13 & 32.5 \\
Physical restraint & 22 & 55.0 \\
\hline
\end{tabular}

Table 6. Secondary outcomes throughout the study period

\begin{tabular}{|lc|}
\hline \multicolumn{1}{|c|}{ Variable } & Mean \pm SD \\
\hline Duration of sedation/analgesia day (Mean \pm SD) & $5.25 \pm 2.18$ \\
Duration of MV days (Mean \pm SD) & $5.32 \pm 2.24$ \\
ICU LOS days (Mean \pm SD) & $6.07 \pm 2.58$ \\
\hline
\end{tabular}

MV: Mechanical ventilation, ICU LOS: Intensive care unit length of stay 


\section{Box 6. Evidence linked analgo-sedation recommendations \\ Serial \\ Recommendation Statements \\ 1. Pain Assessment \\ 1.1. Assess pain for all adult ICU patients regularly. \\ 1.2. Use Behavioral Pain Scale (BPS) or the Critical-Care Pain \\ Observation Tool (CPOT) for pain monitoring. Patient is in significant pain if $\mathrm{BPS}>5$, or CPOT $\geq 3$.}

Grading

B

1.3. Do not use vital signs alone for pain assessment in adult ICU patients.

Vital signs may be used as a cue to begin further pain assessment.

\section{Pain Management}

2.1. Perform non-pharmacologic interventions and/or administer preprocedural analgesia to alleviate pain in adult ICU patients' prior invasive and potentially painful procedures.

2.2. Give fentanyl for acutely distressed patients with hemodynamic instability or renal insufficiency.

2.3. Give morphine for hemodynamically stable patients.

\section{Sedation/agitation Assessment}

3.1. Assess patient's level of sedation regularly

3.2. Use Richmond Agitation-Sedation Scale (RASS) and SedationAgitation Scale (SAS) for sedation assessment.

\section{Sedation/agitation Management}

4.1. Start sedation of agitated mechanically ventilated patients only AFTER providing adequate analgesic and managing reversible physical causes.

4.2. Establish sedation goal to keep patient purposely follows commands without agitation.

4.3. Select analgesic and sedative drugs based upon patient needs, drug allergies, and/or organ dysfunction.

4.4. Use midazolam for rapid sedation of acutely agitated patients and for short-term use only.

4.5. Use propofol for intravenous sedation infusion.

5. 5.1. Sedation protocol/algorithm should be routinely used in adult ICU B patients.

5.2. Use non-pharmacological interventions to promote sleep and increase overall patient comfort. 
Table 7. Appraisers' evaluation of analgo-sedation guidelines quality according to domains' scores of AGREE II instrument

\begin{tabular}{|c|c|c|c|c|c|}
\hline \multirow[b]{2}{*}{ AGREE II Domains } & \multicolumn{5}{|c|}{ Appraisers' Quality Scores \% } \\
\hline & $\begin{array}{l}\text { Academic } \\
\text { Nurses } \\
\mathbf{n}=7\end{array}$ & $\begin{array}{c}\text { Academic } \\
\text { Physicians } \\
\quad n=4\end{array}$ & $\begin{array}{c}\text { Practitioner } \\
\text { Nurses } \\
n=4\end{array}$ & $\begin{array}{c}\text { Practitioner } \\
\text { Physicians } \\
\text { n=3 }\end{array}$ & $\begin{array}{c}\text { Total \% } \\
\text { for All Appraisers } \\
\mathbf{n = 1 8}\end{array}$ \\
\hline Scope and purpose & 92.8 & 84.7 & 79.2 & 92.6 & 87.3 \\
\hline Stakeholder involvement & 84.5 & 83.3 & 79.2 & 83.3 & 82.6 \\
\hline Rigor of development & 93.2 & 72.9 & 80.2 & 76.4 & 80.7 \\
\hline Clarity and presentation & 85.7 & 70.8 & 77.8 & 79.6 & 78.5 \\
\hline Applicability & 83.9 & 76.0 & 81.3 & 84.7 & 81.5 \\
\hline Editorial independence & 82.1 & 70.8 & 79.2 & 83.3 & 78.8 \\
\hline Overall Domains Score\% & 87.0 & 76.4 & 79.5 & 83.3 & 81.6 \\
\hline
\end{tabular}

Table 8. Appraisers' opinion of the overall quality of the developed analgo-sedation guidelines

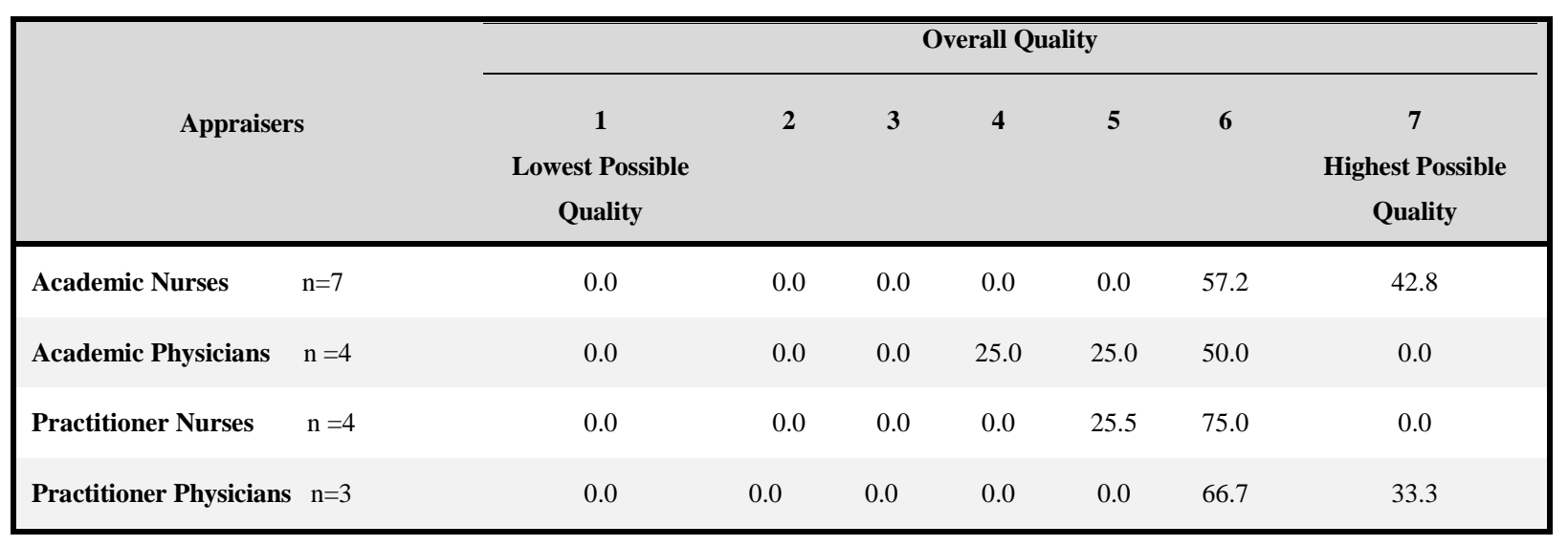

Table 9. Appraisers' recommendations for the use of analgo-sedation guidelines

\begin{tabular}{|c|c|c|c|c|c|c|}
\hline \multirow{2}{*}{ Appraisers } & \multicolumn{2}{|c|}{$\begin{array}{l}\text { Recommended } \\
\text { for Use }\end{array}$} & \multicolumn{2}{|c|}{$\begin{array}{c}\text { Recommended } \\
\text { with Modifications }\end{array}$} & \multicolumn{2}{|c|}{$\begin{array}{c}\text { Not Recommended } \\
\text { for Use }\end{array}$} \\
\hline & Frequency & $\%$ & Frequency & $\%$ & Frequency & $\%$ \\
\hline Academic nursing staff $\quad(n=7)$ & 5 & 71.4 & 2 & 28.6 & 0 & 0.0 \\
\hline Academic medical staff $\quad(n=3)$ & 2 & 66.6 & 1 & 33.3 & 0 & 0.0 \\
\hline Practitioner nurses $\quad(n=4)$ & 4 & 100 & 0 & 0.0 & 0 & 0.0 \\
\hline Practitioner physicians $\quad(n=3)$ & 3 & 100 & 0 & 0.0 & 0 & 0.0 \\
\hline
\end{tabular}

